\title{
Relation Between Body Surface Mapping and Endocardial Spread of Ventricular Activation in Postinfarction Heart
}

\author{
PASCAL F.H.M. VAN DESSEL, M.D., ${ }^{*}$ NORBERT M. VAN HEMEL, M.D., Ph.D.,* \\ JACQUES M.T. DE BAKKER, Ph.D., $\dagger+$ ANDRÉ C. LINNENBANK, Ph.D., $\dagger$ \\ MARK POTSE, M.Sc., $§$ EMILE R. JESSURUN, M.D.,* \\ ARNE SIPPENSGROENEWEGEN, M.D., Ph.D.,II and ERIC F.D. WEVER, M.D., Ph.D.*
}

From the *Department of Cardiology, St. Antonius Hospital, Nieuwegein, The Netherlands; †Experimental and Molecular Cardiology Group of the Cardiovascular Research Institute, University of Amsterdam, Amsterdam, The Netherlands; $¥$ Interuniversity Cardiology Institute of the Netherlands, Utrecht, The Netherlands; the §Department of Medical Physics, Academic Medical Center, Amsterdam, The Netherlands; and I[Section of Cardiac Electrophysiology, Department of Medicine and The Cardiovascular Research Institute, University of California, San Francisco, California

\begin{abstract}
Body Surface Mapping and Endocardial Activation Patterns. Introduction: Body surface mapping (BSM) can be used to identify the site of earliest endocardial activation of ventricular tachycardias (VTs). The multielectrode QRS morphology during VT is determined by both the site of earliest activation and the subsequent spread of electrical activation through the ventricles. This study investigated the relationship between the site of earliest endocardial activation, endocardial spread of activation, and the morphology of the multielectrode surface map in patients with remote myocardial infarction.

Methods and Results: In 14 patients with VT late (8.2 \pm 5.2 years) after myocardial infarction, BSM and simultaneous left ventricular 64-site basket endocardial mapping was performed during a total of 17 monomorphic VTs. In addition, multisite pacing by sequential use of the 64 basket electrodes was performed in 9 patients. BSM and basket mapping revealed the same endocardial breakthrough sites in $8(47 \%)$ of 17 VTs and $189(59 \%)$ of 322 pacing sites; adjacent sites were found in $2(12 \%)$ of 17 VTs and $36(11 \%)$ of 322 pacing sites. Large zones of conduction block explained the mismatch in localization in $2(12 \%)$ of 17 VTs and $52(16 \%)$ of 322 pacing sites. Regional differences in endocardial electrogram amplitudes were found as a cause for dissimilarity in $3(18 \%)$ of 17 VTs and 73 (23\%) of 322 pacing sites. Multiple endocardial breakthrough sites were found in $1(6 \%)$ of 17 VTs and $8(2 \%)$ of 322 pacing sites Finally, an epicardial exit site was suggested in $3(18 \%)$ of 17 VTs as an explanation for mismatch, as no early endocardial activity could be recorded.

Conclusion: Zones of conduction block, regional differences in signal amplitude, and multiple endocardial breakthrough sites are frequent causes for mismatch between BSM and basket catheter activation mapping. (J Cardiovasc Electrophysiol, Vol. 12, pp. 1232-1241, November 2001)
\end{abstract}

body surface mapping, myocardial infarction, ventricular tachycardia, basket mapping

\section{Introduction}

Body surface mapping (BSM) can be used as a noninvasive tool to determine the site of earliest endocardial activation of postinfarction ventricular tachycardia (VT). For this purpose, integral maps of the total QRS complex acquired with multiple body surface leads are compared with a reference database of QRS integral maps previously obtained during endocardial pace mapping. ${ }^{1}$

It is well known that the mechanism underlying postinfarction VT can be attributed to reentry and that the arrhythmogenic substrate often is situated in the subendocardium. ${ }^{2-4}$ The endocardial exit site, i.e., the site where surviving strands of myocardial fibers are connected to the noninfarcted myocardium, can be considered as an electrophysiologically well-defined anatomic landmark of the arrhythmogenic substrate. This landmark can be used as a

Address for correspondence: P.F.H.M. van Dessel, M.D., Department of Cardiology, St. Antonius Hospital, Koekoekslaan 1, 3430 EM Nieuwegein, The Netherlands. Fax: 31-30-603-44-20; E-mail: p.van.dessel@ deskart.n1

Manuscript received 21 June 2001; Accepted for publication 24 September 2001. starting point to identify other parts of the reentrant circuit, such as the central common pathway, which often is used as a direct target for radiofrequency or surgical ablation. ${ }^{5}$

Because of its greater spatial resolution, BSM has been shown to be superior to the conventional 12-lead ECG for assessment of the VT exit site. ${ }^{6,7}$ However, the potential at every point on the body surface is determined by summation of the electric activity in the entire heart. This implies that not only the site of earliest endocardial and epicardial activation, but also the global spread of activation, will contribute to genesis of the QRS complex on the surface ECG. In the structurally normal left ventricle, BSM is capable of providing a high spatial VT localization resolution. ${ }^{5}$ In patients with prior myocardial infarction, the spread of activation during VT may be profoundly affected due to structural and functional changes, which may cause a spatial mismatch between the endocardial and epicardial breakthrough site. ${ }^{8}$ This may partly explain why BSM is able to assess the exact endocardial exit site of postinfarction VT in only $60 \%$ to $70 \%$ of cases whereas it offers an approximation of the origin in an additional $25 \%$ of cases. ${ }^{1}$

To date, there have been no clinical studies reported in which the total body surface QRS morphology was simul- 
taneously compared with the endocardial spread of left ventricular activation. The aim of this study was to identify mechanisms that may cause BSM to fail in assessing the correct site of earliest endocardial activation in patients with prior myocardial infarction. To reach this goal, we simultaneously recorded 62 body surface potentials and 64 endocardial unipolar left ventricular electrograms using a basket catheter in patients with VT remote after myocardial infarction. The endocardial breakthrough site and the BSM-determined site of origin were compared during VT and endocardial pacing. These data were evaluated with respect to the patterns of left ventricular activation.

\section{Methods}

\section{Patient Selection}

All patients admitted to our hospital between November 1997 and February 1999 for antiarrhythmic surgery or radiofrequency catheter ablation of infarct-related VT were considered for inclusion in this study. Specific eligibility criteria included (1) documented recurrent ventricular arrhythmias with or without aborted sudden cardiac death, and (2) inducible sustained monomorphic VT during programmed electrical stimulation. Transthoracic echocardiography was applied to assess the dimensions of the left ventricle and to exclude the presence of a mural left ventricular thrombus or significant aortic valve disease, because these conditions can interfere with safe deployment of the basket catheter. All antiarrhythmic drugs were discontinued for at least five drug elimination half-lives before the study. The research protocol was approved by the Ethical Committee of the St. Antonius Hospital. Prior written informed consent was obtained.

\section{Electrophysiologic Study}

Induction of VT was carried out using a routine stimulation protocol including delivery of up to three extrastimuli following a drive train of eight stimuli (2-msec pulse width at twice diastolic threshold current) with three different cycle lengths $(600,500$, and $430 \mathrm{msec})$. A 6-French quadripolar catheter was positioned at the right ventricular apex or right ventricular outflow tract for this purpose.

If VT proved to be inducible, a 64-electrode unipolar basket catheter (Constellation Catheter; ${ }^{\text {TM }}$ Boston Scientific, Inc., Natick, MA, USA) was inserted percutaneously after termination of VT. In a subset of nine patients, pace mapping during sinus rhythm with the basket catheter was performed before renewed induction of VT was attempted. The threshold was determined separately for each electrode of the basket catheter and, if capture could be obtained, pacing just above the current threshold with a drive cycle of $500 \mathrm{msec}$ was performed.

\section{Data Acquisition and Mapping}

Data from the endocardial basket catheter and BSM electrodes were recorded simultaneously on a battery-powered 128-channel digital acquisition device. 9,10 Analog-todigital conversion occurred at a sampling frequency of 2 $\mathrm{kHz}, 16$-bit resolution, and bitstep of $2 \mu \mathrm{V} / \mathrm{bit}$. Wilson's central terminal was used as reference for unipolar surface ECGs. The amplifier system had a bandpass characteristic using a frequency range from 0.16 to $400 \mathrm{~Hz}$ (3-dB fre-

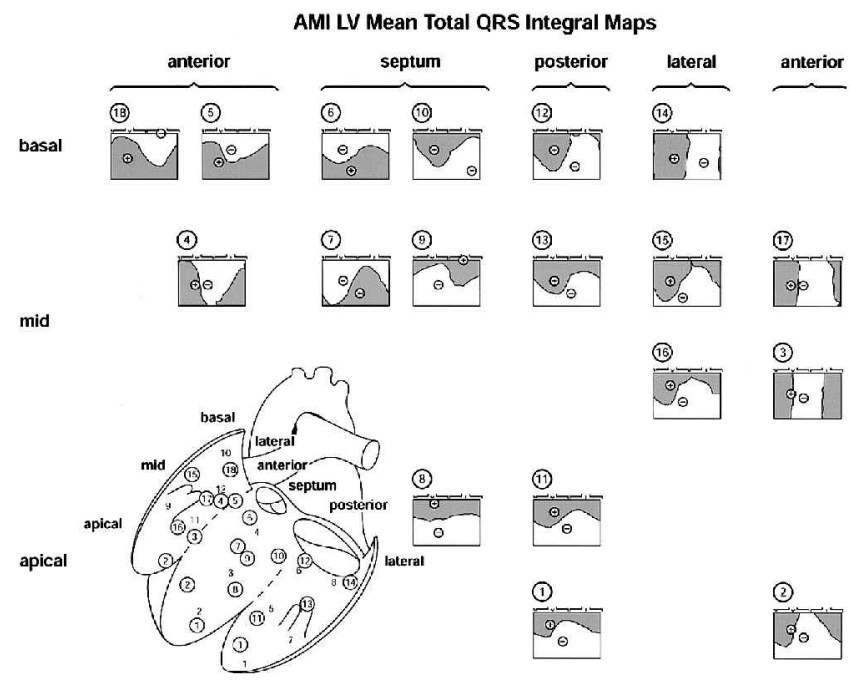

Figure 1. Database of mean paced QRS integral maps previously acquired in patients with prior anterior myocardial infarction (AMI). Encircled numbers depict the location of pacing segments in a schematic overview of the endocardial surface of the left ventricle $(L V)$ as defined in the previously developed AMI (18 segments) database. Mean paced QRS integral database maps are displayed and matched to the endocardial segment by the encircled number. Endocardial segments as defined by Josephson et al. ${ }^{19}$ are indicated by the uncircled numbers (12 segments). (Reproduced with permission from the American Heart Association. ${ }^{13}$ )

quencies). Data were transmitted using a fiberoptic transmission unit and stored in a Pentium-based PC.

\section{Body Surface Mapping}

Body surface ECGs were recorded using a radiotransparent carbon unipolar electrode grid that does not interfere with fluoroscopic imaging during catheterization. The 62 electrodes were positioned in 14 flexible vertical straps that were applied on the anterior and posterior thoracic surfaces, as described elsewhere. ${ }^{11} \mathrm{~A}$ mean of $2.0 \pm 1.8$ leads per map were rejected because of unsatisfactory signal quality and were replaced by a value computed from neighboring electrodes. Our methods of processing and analysis were described previously. ${ }^{12}$ In short, after performing a baseline correction to exclude any possible contribution of baseline drift or interelectrode offset difference, the beginning and end of the QRS complex were set at the time instant at which one of the extreme amplitudes reached $>0.2 \mathrm{mV}$ and at the J point, respectively. A QRS integral map was computed for each VT and for every paced ventricular complex. Each VT and paced QRS integral map pattern was correlated with an anterior or inferior myocardial infarction database comprising mean paced QRS integral maps produced previously at 18 and 22 different specific endocardial segments, respectively (Figs. 1 and 2). ${ }^{13}$ Pattern matching of QRS integral maps was performed both visually and mathematically using correlation coefficients. ${ }^{13,14}$ Visual analysis involved comparison of the position and orientation of the extremes and the morphology of the zero line.

\section{Multielectrode Endocardial Mapping}

\section{Catheter placement}

The basket catheter consists of eight self-expanding nitinol splines mounted on a 110-cm long, 8-French catheter 


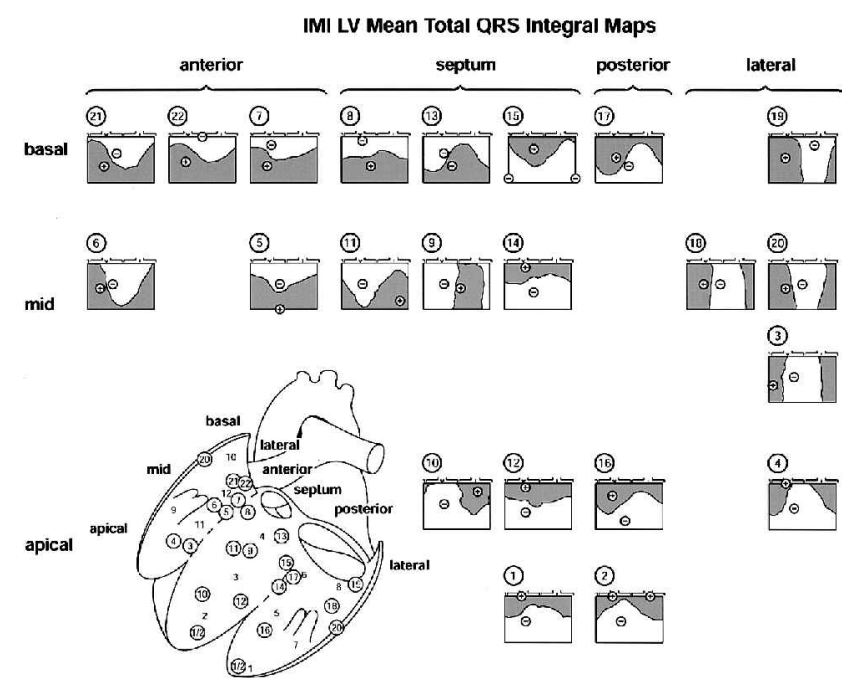

Figure 2. Database representing mean paced QRS integral maps previously acquired in patients with prior inferior myocardial infarction (IMI). Encircled numbers depict the location of pacing segments in a schematic overview of the endocardial surface of the left ventricle $(L V)$ as defined in the previously developed IMI (22 segments) database. Mean paced QRS integral database maps are displayed and matched to the endocardial segment by the encircled number. Endocardial segments as defined by Josephson et al..$^{19}$ are indicated by the uncircled numbers (12 segments). (Reproduced with permission from the American Heart Association. ${ }^{13}$ )

shaft. Each spline has eight symmetrically arranged electrodes. Selection of catheter size was based on the dimensions of the left ventricle as determined by two-dimensional transthoracic echocardiography. In our series, a basket catheter with a diameter of either 75 or $94 \mathrm{~mm}$ was chosen, resulting in a vertical interelectrode spacing of 7 or $9 \mathrm{~mm}$, respectively. Horizontal interelectrode distance varied with positioning and deployment of the catheter. An 11-French introducer was inserted in the right femoral artery using the standard Judkins technique to advance a 7-French pigtail catheter into the left ventricle. Then, a long 10-French guiding sheath was placed in the left ventricle across the aortic valve over the pigtail catheter, and the collapsed basket catheter was advanced through the guiding sheath in the left ventricle after removal of the pigtail catheter. The guiding sheath was pulled back, allowing deployment of the basket catheter in the left ventricle. The activated clotting time was kept longer than 300 seconds by full heparinization to avoid thromboembolic complications. A 6-French quadripolar catheter was inserted percutaneously through the right femoral vein and positioned in the intra-abdominal part of the inferior caval vein to serve as a far-field recording and pacing reference electrode for the unipolar basket catheter electrodes.

\section{Fluoroscopic localization of the basket catheter}

Because basket catheter positioning varies from patient to patient, the exact position of the individual basket electrodes was determined using biplane right anterior oblique (RAO) and left anterior oblique (LAO) digital cine fluoroscopy (Philips Medical Systems Integris, Best, The Netherlands). After selecting the same end-diastolic frames from both fluoroscopic projections, splines A and B, which carry one and two additional radiopaque markers, respectively, were identified (Fig. 3A). After labeling electrodes of these two splines, the other splines could be identified by comparing their relative position with the known position of the first two splines. Each individual electrode and the central apical spline connector were marked and given two-dimensional coordinates in the RAO and LAO projections. A panel of three experienced interventional electrophysiologists verified correct identification of each electrode. Finally, by combining the data from the RAO and LAO projections, three-dimensional coordinates for each electrode were calculated, thereby allowing realistic anatomic rendering of the position and shape of the basket catheter in the left ventricle for each individual patient (Fig. 3B). To be able to compare basket catheter activation mapping and
A
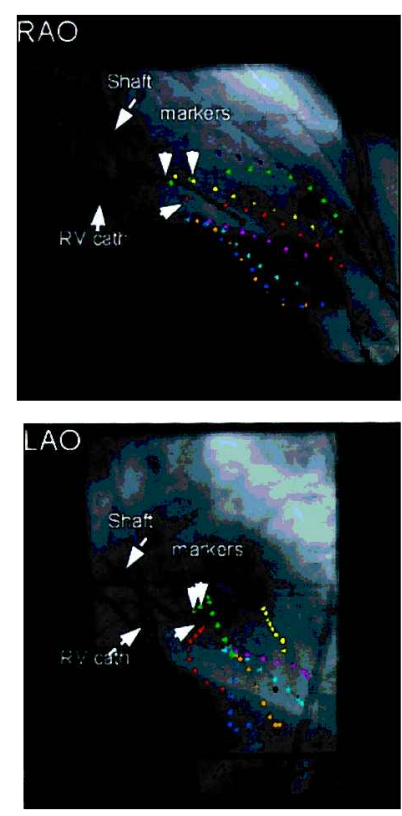

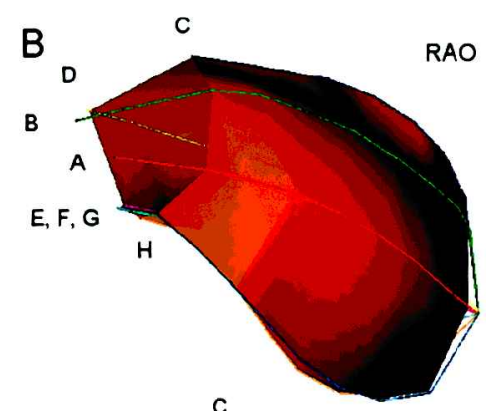

C

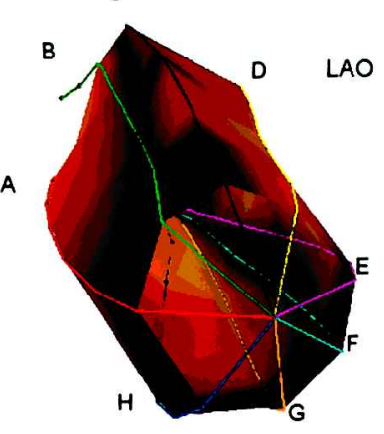

Figure 3. (A) Right (RAO) and left (LAO) anterior oblique fluoroscopic projections of the deployed basket catheter in the left ventricle. Markers for the A and B splines are highlighted by small white arrows. The stimulation catheter ( $R V$ cath) is positioned in the right ventricle and marked with a dashed white arrow. The proximal part of the basket catheter (shaft) is located in the aorta and marked with a dashed white arrow. Recording electrodes are depicted with a dot of unique color for each spline. (B) Reconstruction of the three-dimensional shape and positioning of the basket catheter in the left ventricle as viewed in the RAO and LAO projections. Letters A to $H$ identify the eight individual splines. 


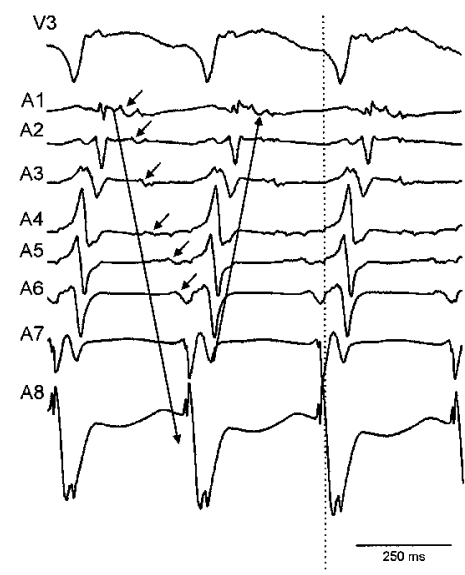

Figure 4. Example of activation sequences reconstructed during ventricular tachycardia (VT). Shown are standard precordial lead V3 and eight endocardial electrograms (A1 to A8) obtained using coaxial electrogram reconstruction. Dotted line indicates the body surface QRS onset. Diastolic activation pattern is indicated by the large downward-pointing black arrow. Small black arrows indicate the presence of diastolic potentials. Diastolic activation leads to electrode A7, where local activation is sharp and initially negative and precedes the onset of the body surface QRS complex. This site is considered the exit site of VT. From there, systolic activation (large upward-pointing black arrow) can be traced back to the start of the diastolic wavefront in electrode A1, thereby completing the reentrant circuit.

pace mapping data with ECG localization results, the location of each basket catheter electrode was mapped to an endocardial segment in one of the two databases of paced QRS integral maps.

\section{Endocardial data processing}

A software package was developed for on-line data processing based on MatLab 5.1 (The MathWorks, Inc., Natick, MA, USA). ${ }^{15}$ The surface Laplacian has been proposed to optimize the detection of small deflections in the endocardial unipolar electrograms. These small sharp deflections are believed to result from local events as opposed to larger, more flaccid, deflections, which are presumed to be caused by remote events. 16-18 The Laplacian suppresses remote deflections, but still has the characteristics of the unipolar electrogram. In contrast to bipolar electrograms, the Laplacian is direction independent. However, the surface Laplacian has only been validated for spatially dense electrode grids (interelectrode distances $\leq 1 \mathrm{~mm}$ ), and no data on its value for electrode systems with interelectrode systems of 5 to $10 \mathrm{~mm}$ are available at present. Therefore, a coaxial electrogram was used in our study. This coaxial electrogram was constructed by subtracting the mean electrogram from the eight surrounding electrodes weighted for distance, from the unipolar signal recorded at the central electrode. This procedure is numerically equivalent to the surface Laplacian but differs from it by a factor that is determined by the electrode distances. Therefore, activation time parameters, such as zero line crossing, moment of $\mathrm{dV} / \mathrm{dt}_{\mathrm{min}}$, and onset and offset of the electrogram are equal to those derived from a surface Laplacian. An example of activation sequences reconstructed with the aid of coaxial electrograms is shown in Figure 4.

\section{Definitions of Parameters and Related Terminology}

VT was considered to be monomorphic and sustained if it lasted for at least 30 seconds without significant changes in QRS morphology as monitored by 12-lead ECG. The local endocardial activation time was determined by the steepest deflection $\left(\mathrm{dV} / \mathrm{dt}_{\mathrm{min}}\right)$ in the local electrogram. An endocardial breakthrough was defined as an endocardial site where an activation wavefront emerged and from where it spread radially. The endocardial VT exit site was defined as the site where the earliest endocardial breakthrough occurred relative to the onset of the QRS complex on the simultaneously recorded surface ECG. ${ }^{19}$ An epicardial breakthrough was defined as the absence of endocardial activity before or within $10 \mathrm{msec}$ after the onset of the body surface QRS complex. If activation times of signals recorded at contiguous sites (7 to $9 \mathrm{~mm}$ apart) differed by 50 msec, conduction block was considered present. ${ }^{20}$

\section{Statistical Analysis}

All data were stored in a computerized patient record database. Data analysis was performed on a personal computer using the SAS System for Windows 6.12 statistical software package (SAS Institute, Cary, NC, USA). Data are given as mean $\pm \mathrm{SD}$, unless stated otherwise. $\mathrm{P}<0.05$ was considered statistically significant.

\section{Results}

\section{Patient Characteristics}

Seventeen consecutive patients with documented infarctrelated sustained ventricular arrhythmias were eligible for the study. In $2(12 \%)$ of 17 patients, no adequate deployment of the basket catheter could be obtained, whereas in 1 (6\%) of 17 patients, only nonsustained VTs could be induced. These 3 patients were excluded from further analysis. The characteristics of the remaining 14 patients are listed in Table 1.

TABLE 1

Baseline Patient Characteristics $(\mathrm{n}=14)$

\begin{tabular}{lc}
\hline Age (years) & $70.1 \pm 6.2$ \\
Sex & 10 \\
$\quad$ Male & 4 \\
Female & $1.3(1-4)$ \\
No. of VT morphologies per patient & $318 \pm 65$ \\
$\quad$ VT cycle length (msec) & $27.8 \pm 9.5$ \\
Scintigraphic LVEF (\%) & \\
Echocardiographic dimensions & $90.5 \pm 9.6$ \\
$\quad$ LV end-diastolic length (mm) & $55.2 \pm 6.8$ \\
LV end-diastolic width (mm) & $3.2(3-5)$ \\
Wall-motion score (maximum 9 of 9 normal segments) & \\
Extent of CAD (>70\% stenosis) & 5 \\
1 vessel & 4 \\
2 vessel & 5 \\
3 vessel & $8.2 \pm 5.2$ \\
Time since infarction (years) & 9 \\
Site of myocardial infarction & 5 \\
$\quad$ Anterior & \\
Inferior &
\end{tabular}

Values are given as mean $\pm \mathrm{SD}$ or median (range).

$\mathrm{CAD}=$ coronary artery disease; $\mathrm{LV}=1$ left ventricle; $\mathrm{LVEF}=1 \mathrm{eft}$ ventricular ejection fraction; VT $=$ ventricular tachycardia. 
TABLE 2

Comparison of VT Site of Origin Determined Using Body Surface Mapping and Basket Activation Mapping

\begin{tabular}{|c|c|c|c|c|c|c|c|}
\hline $\begin{array}{l}\text { Pt. } \\
\text { No. }\end{array}$ & Rhythm & $\begin{array}{c}\text { VT } \\
\text { Morphology }\end{array}$ & Axis & $\begin{array}{l}\text { Cycle Length } \\
\text { (msec) }\end{array}$ & $\begin{array}{c}\text { Segment of Earliest } \\
\text { Endocardial Activation } \\
\text { by BSM }\{\mathrm{JL}\}\end{array}$ & $\begin{array}{c}\text { Site of Earliest Endocardial } \\
\text { Activation by Basket } \\
\text { Catheter }\{\mathbf{J L}\}\end{array}$ & $\begin{array}{c}\text { Distance Between } \\
\text { Suggested } \\
\text { Endocardial } \\
\text { Segments }\end{array}$ \\
\hline 2 & VT1 & RBBB & Inferior & 430 & IMI7 $\{12\}$ & IMI19 $\{10\}$ & Remote \\
\hline \multirow[t]{2}{*}{3} & VT1 & RBBB & Superior & 200 & IMI9 $\{3\}$ & IMI $20\{7\}$ & Remote \\
\hline & VT2 & RBBB & Inferior & 250 & IMI6 $\{11-12\}$ & IMI6 $\{11-12\}$ & Same \\
\hline 4 & VT1 & RBBB & Superior & 420 & AMI17 $\{9-11\}$ & AMI1 $\{1-7\}$ & Remote \\
\hline 7 & VT1 & RBBB & Superior & 320 & AMI8 $\{2-3\}$ & AMI + AMI13 $\{3\}+\{5-7\}$ & Adjacent \\
\hline 8 & VT1 & RBBB & Inferior & 230 & AMI18 $\{10\}$ & AMI18 $\{10\}$ & Same \\
\hline 9 & VT1 & RBBB & Inferior & 240 & AMI18 $\{10\}$ & AMI7 $\{3\}$ & Remote \\
\hline 10 & VT1 & LBBB & Superior & 260 & AMI9 $\{3\}$ & AMI15 $\{9-10\}$ & Remote \\
\hline 11 & VT1 & RBBB & Superior & 440 & IMI16 $\{5\}$ & IMI3 $\{11\}$ & Remote \\
\hline 12 & VT2 & RBBB & Superior & 320 & AMI17 $\{9\}$ & AMI17 $\{9\}$ & Same \\
\hline
\end{tabular}

$\mathrm{AMI}=$ anterior myocardial infarction database segment; BSM $=$ body surface mapping; $\mathrm{IMI}=$ inferior myocardial infarction database segment; JL $=$ Josephson location; LBBB = left bundle branch block morphology; RBBB = right bundle branch block morphology; VT $=$ ventricular tachycardia .

\section{Correlation of VT Localization Using BSM and Basket Catheter Activation Mapping}

A total of 17 sustained monomorphic VTs were localized using BSM and basket catheter activation mapping (Table 2). In $8(47 \%)$ of 17 VTs, the endocardial exit site was located in the endocardial segment predicted by BSM, whereas in 2 other VTs (12\%), the endocardial exit site was situated in a neighboring segment. Seven $(41 \%)$ of 17 VTs originated from a segment remote from the segment identified by BSM. Therefore, in $10(59 \%)$ of 17 VTs, BSM appeared to correctly localize or regionalize the actual endocardial VT exit site. An example of a matching VT site of origin is shown in Figure 5.

\section{BSM During Endocardial Pace Mapping}

For a median of 30 of 64 electrodes (range 17 to 54), pacing capture could be obtained. This required a mean pacing current strength of $7.9 \pm 6.4 \mathrm{~mA}$. Mean interval between the pacing stimulus and the onset of the body surface QRS complex was $36 \pm 20.4 \mathrm{msec}$. There was no significant difference in the mean stimulus-to-QRS interval between matching and nonmatching pace mappings $(\mathrm{P}=$ 0.8 ). This implied that localization mismatch was not due to breakthroughs that were remote from the pacing site, as can be seen when stimulation occurs within the zone of slow conduction. A total of 322 paced QRS maps (median 46.2, range 17 to 54 per patient) were obtained. Mathematical correlation between each paced QRS integral map and the best matching mean paced QRS integral map from the reference database was $0.87 \pm 0.07$ (range 0.48 to 0.98 ). For $189(59 \%)$ of the 322 paced QRS integral maps, the fluoroscopic position of the pacing electrode was located in the same endocardial segment as the best matching mean paced QRS integral map from the database. An adjacent segment of the database was found in $36(11 \%)$ of 322 pacing sites. The database segment was located remote from the endocardial pacing site in 97 (30\%) of 322 paced QRS integral maps.

\section{Electrophysiologic Mechanisms Responsible for Mapping Mismatches}

Endocardial activation patterns and electrogram distributions acquired during VT and pacing with the basket catheter were reviewed in detail to explain mismatches between sites of earliest endocardial activation identified with BSM and basket mapping. Three major mechanisms could be identified: extensive lines of conduction block, multiple endocardial exit sites, and regional differences in endocardial signal amplitude.

\section{Conduction block}

Substantial lines of conduction block were found in 2 $(12 \%)$ of 17 VTs that were both obtained in patients with inferior myocardial infarction. These lines of block prevented radial spread from the earliest activated endocardial site. In $52(16 \%)$ of 322 paced activation sequences, lines of block prevented radial spread from the area of initial activation. This resulted in a mismatch between the segment of earliest activation determined with BSM and the actual location of the pacing electrode on the basket catheter. At 36 (69\%) of 52 pacing sites that were not correctly localized with BSM, the best matching database segment was situated remote from the pacing electrode, whereas in $16(31 \%)$ of these 52 incorrectly identified pacing sites, the pacing location was localized to an adjacent database segment. An example of BSM mismatch as a result of conduction block is shown in Figure 6.

\section{Multiple endocardial breakthroughs}

In $1(6 \%)$ of 17 VTs that was obtained in a patient with previous anterior myocardial infarction (patient 7 in Table 2), two endocardial breakthroughs were found: one in the 
Figure 5. Endocardial activation mapping and body surface mapping during ventricular tachycardia (VT) in patient 6 (Table 2), who had a previous anterior infarction. (A) Activation sequence of the left ventricle in right (RAO) and left (LAO) anterior oblique projections. Activation times in the isochrone maps are relative to onset of the body surface QRS complex and range from $2 \mathrm{~ms}$ (red) to $268 \mathrm{~ms}$ (blue). The VT exit site is located mid-septal at electrode A3 (origin of gray arrows). (B) VT QRS integral map, which is compatible with the mean paced QRS integral map belonging to segment AMI9 (correlation coefficient of 0.92) located in the midseptal wall, compatible with Josephson location 3.

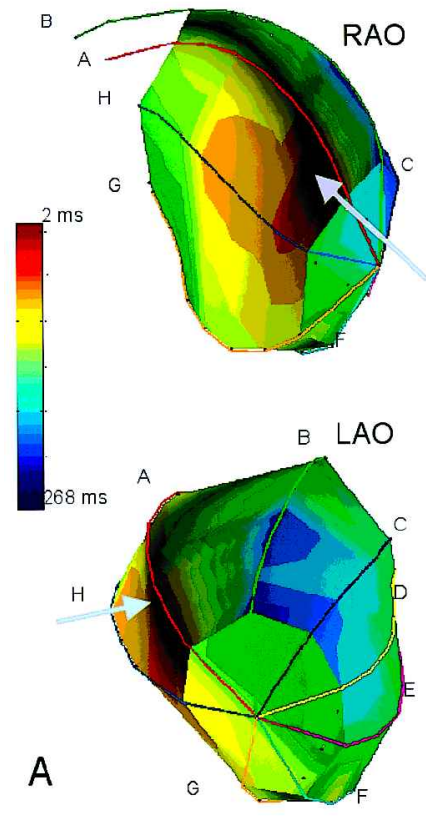

$V T, r=0.92$

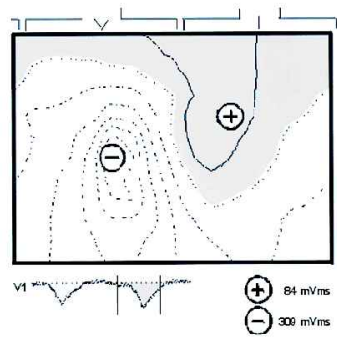

B mid-anteroseptal area and the other near the posterior papillary muscle. Localization using the BSM database indicated a mid-inferoseptal segment of origin, which is located between the two actual endocardial breakthrough sites.

During pace mapping, multiple breakthrough sites (i.e., two distinct sites with radial spread of activation) were found in $8(2 \%)$ of 322 maps. In these cases, the site of stimulation appeared to be at a remote location with respect to the database segment identified with BSM. An example is shown in Figure 7.

\section{Differences in signal amplitude}

In $3(18 \%)$ of 17 VTs where the BSM database localized the site of origin to an adjacent endocardial segment, the electrogram amplitude at the endocardial segment of origin predicted by BSM was much larger than that measured at the true endocardial exit. At 73 (23\%) of 322 pacing sites, differences in signal amplitude resulted in a mismatch between the two methods. The obtained database segment was remote in $52(71 \%)$ of 73 incorrectly localized pacing sites. At $21(29 \%)$ of 73 pacing sites, an adjacent database segment was predicted. An example of BSM mismatch caused by differences in magnitude of the endocardial potential is shown in Figure 8.

\section{Epicardial breakthrough}

In $3(18 \%)$ of $17 \mathrm{VTs}$, no clear reason for mismatch could be found by examining the endocardial spread of activation. Two of these three VTs were documented in patients with previous anterior myocardial infarction, and
Figure 6. Endocardial activation mapping and body surface mapping during ventricular tachycardia (VT) in patient 2 (Table 2), who had a previous posterolateral infarction. (A) Activation sequence of the left ventricle in right (RAO) and left (LAO) anterior oblique projections. Activation times in the isochrone maps are relative to onset of the body surface QRS complex and range from $-9 \mathrm{~ms}$ (red) to $127 \mathrm{~ms}$ (blue). The VT exit site is located basal and posterolateral at electrode $A 7$ (origin of white arrows). A line of block extending from the basal posterolateral area to the apical posterolateral area (along spline B) can be noted (white dotted line). This line of block forces the activation front to propagate toward the anterolateral wall. (B) RAO basket projection has been mirrored computationall y in horizontal and vertical directions to provide a nonstandard rear projection to demonstrate the area of interest, i.e., the line of block along the B spline. (C) VT QRS integral map, which is compatible with the mean paced QRS integral map belonging to segment IMI7 (correlation coefficient of 0.91) located at a remote segment in the basal anterior wall compatible with electrode 1 on spline G (Josephson location 12).
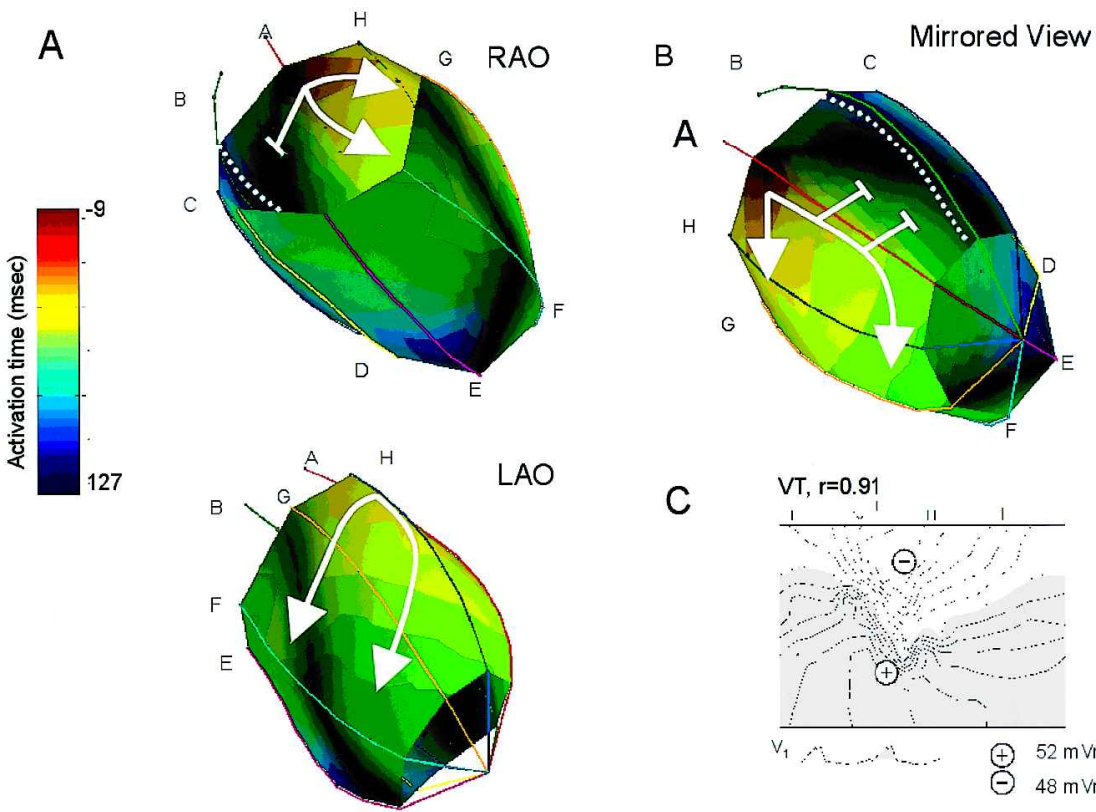

C

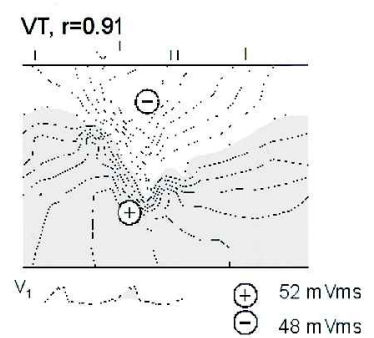




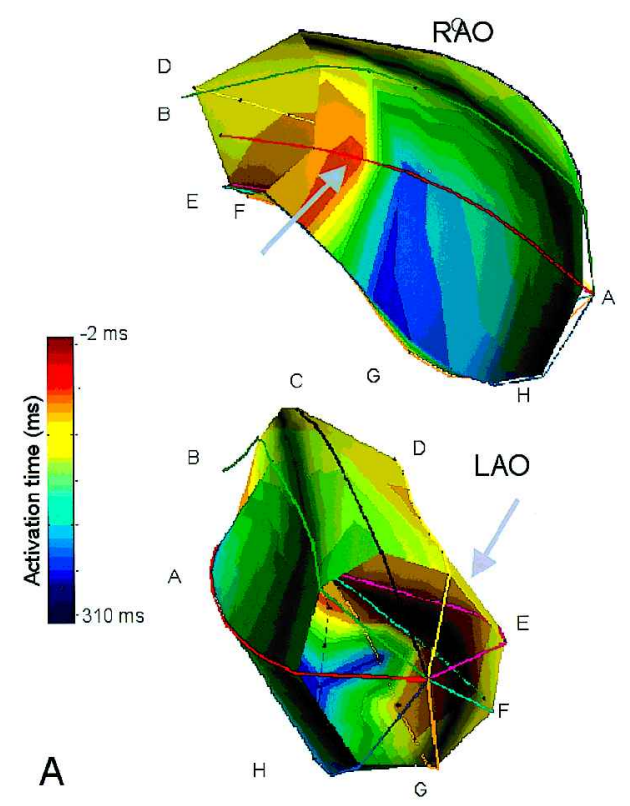

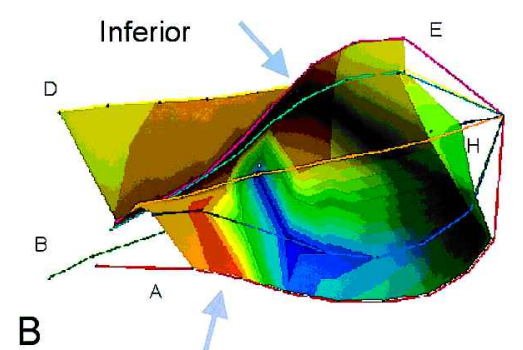

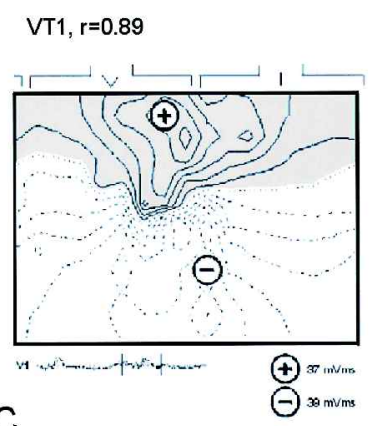

Figure 7. Endocardial activation mapping and body surface mapping during ventricular tachycardia (VT) in patient 7 (Table 2), who had a previous anterior infarction. (A) Activation sequence of the left ventricle in right (RAO) and left (LAO) anterior oblique projections. Activation times in the isochrone maps are relative to onset of the body surface QRS complex and range from $-9 \mathrm{~ms}$ (red) to $127 \mathrm{~ms}$ (blue). The $V T$ exit site is located anteroseptal and midposterior at electrode A7 and electrode F5 (origin of gray arrows). (B) Basket projection has been rotated computationally to provide a nonstandard inferior view. Clearly, two distinct exit sites are visible. (C) VT QRS integral map, which is compatible with the mean paced QRS integral map belonging to segment AMI8 (correlation coefficient of 0.89 ) located at an adjacent segment in the inferoseptal region, compatible with electrode 4 on spline $H$ (Josephson location 3-5). one VT was found in a patient with a prior inferior myocardial infarction. In these VTs, no early endocardial activity (i.e., within $10 \mathrm{msec}$ after the onset of the body surface QRS) was noted. These findings were considered to be suggestive of an epicardial or intramural exit.

\section{Discussion}

The present study shows that BSM indicated the correct left ventricular segment of initial endocardial activation in $50 \%$ to $60 \%$ of VT episodes and endocardial pacing sequences, whereas in an additional $10 \%$ to $20 \%$, regional approximation was achieved. These localization results are comparable to previously reported retrospective data obtained in a similar patient cohort. ${ }^{1}$
The earliest site of endocardial activation, subsequent spread of ventricular activation, and site of epicardial breakthrough all contribute to determining QRS morphology on the body surface ECG. To predict the earliest site of endocardial activation, one of the assumptions made in ECG is that the activation front during VT spreads out centrifugally and travels predominantly in a perpendicular fashion from the presumed endocardial breakthrough site to the epicardial surface. However, in postinfarction VT, the anatomic substrate often is extensively altered. Therefore, the spread of activation may deviate from its anticipated centrifugal pattern. This can give rise to alternative activation spread patterns after myocardial infarction. ${ }^{8}$ It also has been shown that minor shifts in the endocardial exit site can lead to

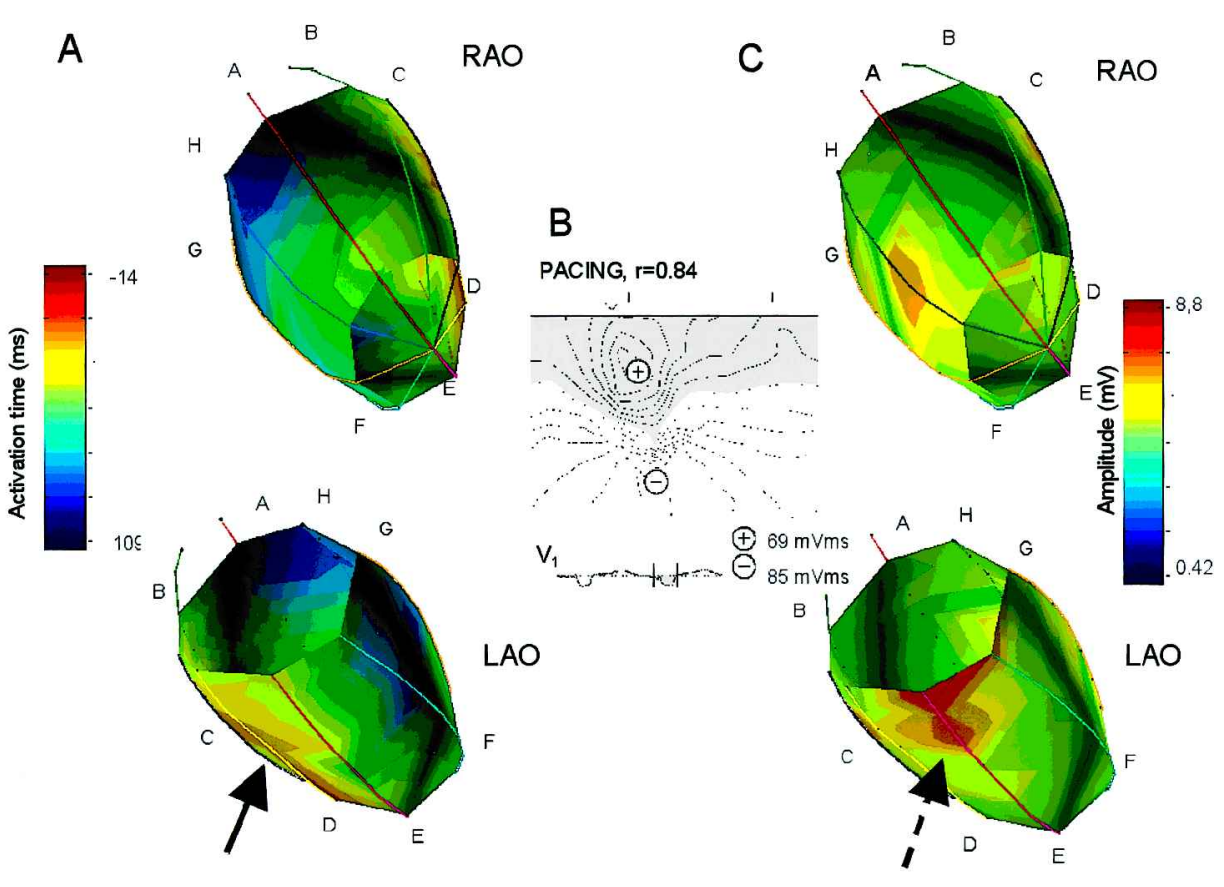

Figure 8. Endocardial pace mapping data obtained in patient 2 , who had a previous posterolateral myocardial infarction. The infarction scar is located basal posterolateral near splines $A$ and B. Stimulation is performed at electrode $C 2$, located midinferior (Josephson location 5). (A) Activation sequence of the left ventricle in right (RAO) and left (LAO) anterior oblique projections, ranging from $-14 \mathrm{~ms}$ at electrode C2 (red) to $109 \mathrm{~ms}$ (dark blue). Solid black arrow indicates the site of stimulation. (B) Paced QRS integral map that compares best (correlation coefficient of 0.84) with the mean paced QRS integral map of database segment IMI14, which is located basal inferoseptal (Josephson location 4-6). (C) RAO and LAO projections of the distribution of endocardial signal amplitude, ranging from 0.42 $\mathrm{mV}$ (dark blue) to $8.8 \mathrm{mV}$ (red). Dashed black arrow indicates the position of database segment IMI14, which is situated adjacent to the stimulation site in an area where the signal amplitude is larger. 
major differences in body surface ECG morphology if transmural spread of activation is not directly from endocardium to epicardium. ${ }^{21-24}$ Although this problem is partially circumvented by using BSM databases that are specific for previous anterior or inferior myocardial infarction, it is conceivable that due to the complex anatomic nature of the postinfarction arrhythmogenic substrate, activation patterns may differ between individual patients. This may lead to incorrect BSM prediction of the presumed endocardial segment of origin of postinfarction VT. In our study, several patterns of activation were identified that can explain disparity in mapping outcome.

\section{Electrophysiologic Mechanisms Causing Disparity Between BSM and Endocardial Mapping}

Nonhomogeneous spread of endocardial activation due to large zones of conduction block caused dissimilarity in mapping findings in 2 (12\%) of 17 VTs and 52 (16\%) of 322 paced activation sequences. Zones of conduction block prevented radial spread of activation from the site of endocardial breakthrough. This also was observed in a canine infarct model where the extent of the line block was one of the causes of different scalar ECG morphologies during sustained VT. ${ }^{25}$ Osswald et al. ${ }^{24}$ showed in a similar experimental model that minor changes in the site of endocardial breakthrough can lead to different activation spread patterns and subsequent body surface ECG morphology .

Two separate endocardial breakthrough sites were found in only a minority of cases during pacing and VT. Multiple breakthrough sites in the postinfarction heart, especially after anterior myocardial infarction, have been reported during both sinus rhythm ${ }^{8}$ and VT. ${ }^{26}$ Because databaseguided BSM of postinfarction VT is developed to localize a single segment of earliest activation, simultaneous occurrence of $\geq 2$ distant breakthrough sites, by definition, will produce an incorrect localization result using the BSM approach. In our study, the segment of VT origin was, however, spatially localized by BSM between the two actual endocardial breakthrough sites.

In $15 \%$ to $20 \%$ of VT episodes or pacing sequences, the breakthrough site was situated in the infarct border generating small-signal amplitudes, whereas signal amplitudes were larger in other noninfarcted endocardial areas. Because high-voltage areas will generate more electromotive force on the surface ECG, they may partially suppress the lowvoltage information resulting from the true endocardial site of VT origin. Mathematical correlation between measured VT QRS integral maps and reference database mean paced QRS integral maps currently is obtained by computing the normalized inner product of the vectorized measured map and all vectorized reference database maps and selecting the highest correlation coefficient. ${ }^{12}$ This makes the computational result more susceptible to the number, location, and magnitudes of the positive and negative extremes on the map. If the magnitude of the positive and negative extremes on the map differ considerably, the best mathematical match between the VT or paced QRS integral map and the reference database mean paced QRS integral map will be based primarily on the position of the larger extreme.

Although the reentrant circuit in postinfarction VT is believed to be located predominantly in the subendocardial layer, other mapping studies that combined endocardial and epicardial mapping have demonstrated that approximately $15 \%$ of VTs have a partially subepicardial circuit and an earlier epicardial breakthrough of the activation wavefront. ${ }^{27}$ We did not find clear early endocardial activity within $10 \mathrm{msec}$ of onset of the body surface QRS complex in $18 \%$ of VTs. In these cases, an earlier epicardial or intramural exit site was believed to be present, although this could not be confirmed due to lack of epicardial activation data. Absence of early endocardial activation also may have been caused by insufficient endocardial basket coverage of the endocardial exit site. However, the incidence of our findings is similar to the incidence of early epicardial breakthrough of postinfarction VT reported in the literature. ${ }^{27}$

\section{Methodologic Considerations in QRS Isointegral Mapping}

Apart from electrophysiologic considerations, methodologic issues connected with reference database-oriented QRS isointegral mapping should be considered as well. Correct identification of the baseline and onset QRS are crucial for accurate computation of QRS areas intended for QRS integral map localization of sites of VT origin. Two major problems with baseline correction and identification of onset of the QRS complex are superposition of onset of the QRS complex on the $\mathrm{T}$ wave of the preceding QRS complex and nonsimultaneous departure of the QRS complex in different leads. Two separate cases should be contemplated. The first case is when the VT under study does not have an extremely short cycle length. Then, usually an isoelectric interval between T wave and QRS onset can be identified, which can be utilized for baseline correction. Onset and offset QRS subsequently are chosen from an instantaneous isopotential map as the instant when the first electrode has reached a value $>0.2 \mathrm{mV}$ (onset) and the instant when each lead has dropped to $<0.2 \mathrm{mV}$ (offset), respectively. In this case, nonsimultaneous departure of the QRS complex will not be of great influence because the integral of the QRS complex is used. The contribution of the integral of a prolonged isoelectric interval before onset QRS in an individual lead with late QRS departure to the entire QRS integral will be approximately zero. It is possible that in leads with earliest QRS complex departure, a small post J-point part of the ST-T segment is integrated, but because voltages in the early ST-T segment usually are low, the integral of these early ST-T segment parts over a small time interval will be negligible compared with the magnitude of the QRS integral.

The second case is when the VT under study has a short cycle length and there is no clear isoelectric interval between the T wave end and onset QRS. VTs of this particular rapid rate usually will be sinusoid in shape with QRS complex and $\mathrm{T}$ wave morphology that are approximately similar but opposite in sign. Baseline correction points subsequently are chosen at best clinical judgment. The possibility remains that-due to confluence of QRS complex and $\mathrm{T}$ wave and nonsimultaneous QRS complex departure-unequal parts of the $\mathrm{T}$ wave are integrated with the QRS complex in different leads. However, in these leads with late QRS departure, unequal parts of the terminal QRS complex will not be included in the QRS integral. This will amount to approximately the same absolute value as the additional area of the $\mathrm{T}$ wave end that was erroneously 
added in these leads because the $\mathrm{T}$ wave has approximately the same shape as the QRS complex. We believe these errors of measurements will approximately annihilate each other, because the QRS complex and T wave are of opposite sign. This will still allow QRS integral map localization by reference database comparison.

\section{Study Limitations}

The basket catheter used in this study is introduced in the left ventricle in a collapsed state. Before it is capable of providing high-resolution mapping of endocardial activation, it has to be fully deployed in the left ventricle. To reliably locate the exit site of VT, adequate endocardial basket catheter coverage of the exit site area is mandatory. Because complete deployment of the catheter is not always feasible, endocardial VT breakthrough sites located in uncovered endocardial regions may have not been recorded. To prevent this, only 14 of 17 patients in whom deployment of the catheter was thought to be adequate were included; 2 patients with imperfect catheter positioning were omitted from further analysis. In addition, localization differences were studied during basket pacing. When endocardial pacing is performed and capture is obtained, the exact site of earliest endocardial activation is the fluoroscopic location of the basket catheter electrode. Therefore, endocardial basket coverage is less an issue during endocardial pacing.

No simultaneous epicardial mapping was performed; therefore, VTs with an epicardial exit site may have been wrongly classified as VTs where the BSM localization and endocardial exit site did not match. In 3 of 17 VTs in our series, no early endocardial breakthrough could be detected; therefore, an epicardial exit was believed to be present.

\section{Clinical Application of BSM}

BSM has been used previously to guide ventricular arrhythmia surgery. In our study, BSM has proven to identify or approximate the segment containing the VT exit site in $70 \%$ to $80 \%$ of cases. Because the surgeon can ablate the reentrant circuit more substantially than the more circumscribed thermal lesion produced during radiofrequency ablation, the resolution offered by BSM appears to be sufficient. 28

In a previous study, BSM has proven to be of great clinical utility in guiding radiofrequency ablation of idiopathic VT. ${ }^{5}$ The mapping results in that study are highly comparable to the localization results in our study. Peeters et al..$^{5}$ compared reference database-guided BSM with endocardial pace mapping in the same patient. The same endocardial segment was found in 53\% of VTs, and $47 \%$ of VTs were mapped to an adjacent endocardial segment. It should be noted that the patients involved in this study did not have any structural heart disease. Therefore, it can be expected that activation spread patterns were not influenced by altered anatomy of the left or right ventricle. Furthermore, when using pace mapping in combination with BSM and comparing the paced maps with the QRS integral map of VT induced in the same patient, as done in the study of Peeters et al., 5 each patient was used as his or her own reference. Because all map comparisons are done in the same patient, i.e., the same electrophysiologic substrate, discrepancies due to differences in torso, heart geometry, and individual activation spread patterns are no longer relevant.

Reference database-guided BSM is a noninvasive tool that can greatly facilitate mapping of postinfarction VT, including hemodynamic unstable and nonsustained VTs. In the normal left ventricle, BSM offers high spatial mapping resolution that is appropriate to directly guide radiofrequency ablation. However, changes in activation spread patterns in the structurally altered postinfarction heart cause BSM to provide inaccurate results in $20 \%$ to $30 \%$ of the localized VTs.

\section{References}

1. SippensGroenewege n A, Spekhorst H, van Hemel NM, Kingma JH, Hauer RN, de Bakker JM, Grimbergen CA, Janse MJ, Dunning AJ: Value of body surface mapping in localizing the site of origin of ventricular tachycardi a in patients with previous myocardial infarction. J Am Coll Cardiol 1994;24:1708-1724

2. Wellens HJ, Durrer D, Lie KI: Observations on mechanisms of ventricular tachycardia in man. Circulation 1976;54:237-244 .

3. Josephson ME, Horowitz LN, Farshidi A, Kastor JA: Recurrent sustained ventricular tachycardia. I. Mechanisms. Circulation 1978;57: 431-440.

4. Stevenson WG, James NW, Wiener I, Nademanee K: Slow conduction in the infarct scar: Relevance to the occurrence, detection and ablation of ventricular reentry circuits resulting from myocardial infarction. Am Heart J 1989;117:452-467.

5. Peeters HA, SippensGroenewegen A, Wever EF, Ramanna H, Linnenbank AC, Potse M, Grimbergen CA, van Hemel NM, Hauer RN, Robles de Medina EO: Clinical application of an integrated 3-phase mapping technique for localization of the site of origin of idiopathic ventricular tachycardia. Circulation 1999;99:1300-1311.

6. SippensGroenewege n A, Spekhorst H, van Hemel NM, Kingma JH, Hauer RN, de Bakker JM, Grimbergen CA, Janse MJ, Dunning AJ: Localization of the site of origin of postinfarction ventricular tachycardia by endocardial pace mapping. Body surface mapping compared with the 12-lead electrocardiogram. Circulation 1993;88:2290-2306.

7. Kuchar DL, Ruskin JN, Garan H: Electrocardiographi c localization of the site of origin of ventricular tachycardia in patients with prior myocardial infarction. J Am Coll Cardiol 1989;13:893-903

8. Hatala R, Savard P, Tremblay G, Page P, Cardinal R, Molin F, Kus T, Nadeau R: Three distinct patterns of ventricular activation in infarcted human hearts. An intraoperative cardiac mapping study during sinus rhythm. Circulation 1995;91:1480-1494

9. Grimbergen CA, Metting van Rijn AC, Kuiper AP, Linnenbank AC, Peper A: Instrumentation for the recording and digital processing of multichannel ECG data. Proc IEEE Biomed Soc 1992;40:726-727.

10. Metting van Rijn AC, Kuiper AP, Linnenbank AC, Grimbergen CA: Patient isolation in multichannel bioelectric recordings by digital transmission through a single optical fiber. IEEE Trans Biomed Eng 1993;40:302-308

11. SippensGroenewegen A, Spekhorst H, Hauer RN, van Hemel NM, Broekhuijsen P, Dunning AJ: A radiotransparent carbon electrode array for body surface mapping during cardiac catheterization. Proc IEEE Biomed Soc 1987;178-181.

12. Linnenbank AC: On-site recording, analysis, and presentation of multichannel ECG data. Thesis. University of Amsterdam, Amsterdam, The Netherlands, 1996

13. SippensGroenewege n A, Spekhorst H, van Hemel NM, Kingma JH, Hauer RN, Janse MJ, Dunning AJ: Body surface mapping of ectopic left ventricular activation. QRS spectrum in patients with prior myocardial infarction. Circ Res 1992;71:1361-1378

14. SippensGroenewege n A, Spekhorst H, van Hemel NM, Kingma JH, Hauer RN, Janse MJ, Dunning AJ: Body surface mapping of ectopic left and right ventricular activation. QRS spectrum in patients without structural heart disease. Circulation 1990;82:879-896.

15. Potse M, Linnenbank AC, Grimbergen CA: Software design for analysis of multichannel intracardiac and body surface electrocardiograms. Comput Methods Programs Biomed 2001.

16. Huiskamp G: Difference formulas for the surface Laplacian on a triangulated surface. J Comp Phys 1991;95:477-496.

17. Maglaveras N, de Bakker JM, van Capelle FJ, Pappas C, Janse MJ: Activation delay in healed myocardial infarction: A comparison between model and experiment. Am J Physiol 1995;269:H1441-H1449. 
18. Coronel R, Wilms-Schopman FJ, de Groot JR, Janse MJ, van Capelle FJ, de Bakker JM: Laplacian electrograms and the interpretation of complex ventricular activation patterns during ventricular fibrillation. J Cardiovasc Electrophysiol 2000;11:1119-1128 .

19. Josephson ME, Horowitz LN, Farshidi A, Spear JF, Kastor JA, Moore EN: Recurrent sustained ventricular tachycardia. 2. Endocardial mapping. Circulation 1978;57:440-447.

20. Kleber AG, Janse MJ, Wilms-Schopman FJ, Wilde AA, Coronel R: Changes in conduction velocity during acute ischemia in ventricular myocardium of the isolated porcine heart. Circulation 1986;73:189198.

21. Spielman SR, Michelson EL, Horowitz LN, Spear JF, Moore EN: The limitations of epicardial mapping as a guide to the surgical therapy of ventricular tachycardia. Circulation 1978;57:666-670.

22. Spear JF, Michelson EL, Spielman SR, Moore EN: The origin of ventricular arrhythmias 24 hours following experimental anterior septal coronary artery occlusion. Circulation 1977;55:844-852 .

23. Kimber S, Downar E, Harris L, Langer G, Mickleborough LL, Masse S, Sevaptsidis E, Chen TC: Mechanisms of spontaneous shift of surface electrocardiographi c configuration during ventricular tachycardia. J Am Coll Cardiol 1992;20:1397-1404 .
24. Osswald S, Wilber DJ, Lin JL, Du D, Holden HB, Ruskin JN, Garan $\mathrm{H}$ : Mechanisms underlying different surface ECG morphologies of recurrent monomorphic ventricular tachycardia and their modification by procainamide. J Cardiovasc Electrophysiol 1997;8:11-23.

25. Costeas C, Peters NS, Waldecker B, Ciaccio EJ, Wit AL, Coromilas J: Mechanisms causing sustained ventricular tachycardia with multiple QRS morphologies: Results of mapping studies in the infarcted canine heart. Circulation 1997;96:3721-3731 .

26. Harris L, Downar E, Mickleborough L, Shaikh N, Parson I: Activation sequence of ventricular tachycardia: Endocardial and epicardial mapping studies in the human ventricle. J Am Coll Cardiol 1987;10:10401047.

27. Kaltenbrunner W, Cardinal R, Dubuc M, Shenasa M, Nadeau R, Tremblay G, Vermeulen M, Savard P, Page PL: Epicardial and endocardial mapping of ventricular tachycardia in patients with myocardial infarction. Is the origin of the tachycardia always subendocardially localized? Circulation 1991;84:1058-1071.

28. Blanchard SM, Wallcott GP, Wharton JM, Ideker RE: Why is catheter ablation less successful than surgery for treating ventricular tachycardia that results from coronary artery disease? PACE 1994;17:23152335. 